Théologiques

Théologiques

\title{
Pour en finir avec la laïcité (sans retomber dans l'eau bénite)
}

\section{Jean Pichette}

Volume 6, numéro 1, mars 1998

Autres regards sur la laïcité

URI : https://id.erudit.org/iderudit/024954ar

DOI : https://doi.org/10.7202/024954ar

Aller au sommaire du numéro

\section{Éditeur(s)}

Faculté de théologie de l'Université de Montréal

\section{ISSN}

1188-7109 (imprimé)

1492-1413 (numérique)

Découvrir la revue

\section{Citer cet article}

Pichette, J. (1998). Pour en finir avec la laïcité (sans retomber dans l'eau bénite). Théologiques, 6(1), 39-54. https://doi.org/10.7202/024954ar

\section{Résumé de l'article}

Le débat sur la laïcité, parce qu'il s'inscrit dans le cadre du dualisme ontologique légué par le christianisme, s'avère incapable de penser l'auto-institution de la société dans le maintien d'un rapport - profane - à la transcendance. Héritière du positivisme du XIXe siècle, la laïcité bute désormais sur l'aporie de ce qui est présenté comme une pédagogie sociale procédant d'une réification de l'espace social. Au nom du pluralisme, elle nourrit ainsi une hétéronomie croissante de la société, allant de pair avec une gestion technocratique du social. 
Théologiques 6/1 (1998) 39-54.

\title{
Pour en finir avec la laïcité (sans retomber dans l'eau bénite)
}

\author{
Jean PICHETTE \\ Journaliste, Le Devoir \\ Membre du Groupe interuniversitaire \\ d'étude de la postmodernité
}

\section{RÉSUMÉ}

Le débat sur la laïcité, parce qu'il s'inscrit dans le cadre du dualisme ontologique légué par le christianisme, s'avère incapable de penser l'auto-institution de la sociéré dans le maintien d'un rapport - profane - à la transcendance. Héritière du positivisme du XIX siècle, la laïcité bute désormais sur l'aporie de ce qui est présenté comme une pédagogie sociale procédant d'une réification de l'espace social. Au nom du pluralisme, elle nourrit ainsi une hétéronomie croissante de la société, allant de pair avec une gestion technocratique du social.

Because the debate on laicity is inscribed within the framework of ontological dualism, a legacy of Christianity, it appears incapable of thinking the "auto-institution * of society while maintaining a relationship .- profane .. with that which is transcendent. As heir to 19 th century positivism, the laicity comes up against the aporia of that which is presented as social pedagogy arising from the reification of social space. In the name of pluralism, laicity nourishes a growing heteronomy of society, paired with a technocratic management of the social.

Je veux depuis longtemps écrire un oratorio, dont le sujet devrait être le suivant : comment l'homme d'aujourd'hui, qui est passé par le matérialisme, le socialisme, l'anarchie, mais qui a tout de même gardé en lui un petit reste de l'ancienne foi (sous forme de superstition), comment cet homme moderne se querelle avec Dieu et parvient finalement à trouver Dieu et à devenir un être religieux. Apprendre à prier! 


\section{À la mémoire de Julien Harvey}

Dans son oratorio L'échelle de Jacob, qu'il n'a finalement jamais complété, Schönberg reprenait la figure de l'ange, si importante dans l'histoire du christianisme. Peut-être ignorait-il la fortune que l'être ailé connaîtrait en cette fin de millénaire. Il semble en tout cas, à en juger par ce qu'il écrivît au poète Richard Dehmel, qu'il ne s'illusionnait guère sur un éventuel dépassement du religieux : malgré, ou peut-être à cause de la modernité et son cortège rationaliste, il rappelle qu'il faut " apprendre à prier ". C'est sous l'œil - narquois ? - d'un compositeur qui se définissait lui-même comme "un conservateur qu'on a forcé à devenir révolutionnaire » que la question de la laïcité sera ici abordée, sous un angle à dessein provocateur, d'autant plus qu'il est tracé par un agnostique. Pour reprendre une boutade de Schönberg, qui déclara un jour que "personne n'ayant voulu l'être, je me suis porté volontaire" pour composer une musique si dissonante, j'oserai demander si le combat pour la laïcité n'est pas au fond un combat d'arrière-garde, qui appelle d'urgence un changement de perspective dans la façon de penser, et de vivre, notre rapport à la transcendance.

Je le confesse - on n'échappe décidément pas à l'inscription de notre histoire dans celle du christianisme -, le débat sur la laïcité m'ennuie. Pour une raison fort simple : les termes dans lesquels il enferme l'équation laïc/religieux me semblent erronés. Comme si les dés étaient pipés d'avance. Nous aurions d'un côté (à gauche ?) la laïcité, portée par les lumières de la Raison, synonyme de progrès et d'émancipation; de l'autre, la religion, viciée en son essence même par l'aliénation - rappelons-nous "l'opium du peuple " de Marx -, et bornée par un conservatisme impénitent. Dans ce schéma binaire, l'autonomie ne pourrait ainsi loger qu'à l'enseigne de la laïcité, véritable antidote à la dépossession de lui-même à laquelle le genre humain se serait lui-même condamné, en érigeant un miroir divin sans lequel il se serait persuadé ne pouvoir se réfléchir. L'histoire de la laïcité serait dans cette optique celle d'un dévoilement, au propre et au figuré, de la vérité de la souveraineté de l'homme, désormais libéré des oripeaux d'une idéalité qui lui aurait jusque-là échappée en partie.

Mais ainsi appréhendée, cette histoire se déploie tout entière dans le creux de la temporalité. Sans épaisseur ontologique, elle ne prend son sens que dans la négation de son historicité, en oubliant en quelque sorte la dimension intrinsèquement agnostique de la laïcité. À la théologie, elle fait succéder une téléologie profane qui réduit l'histoire à un simple intermède. Une fois les pages de celle-ci tournées, la célébration de la révélation laïque ne peut alors masquer que le problème de la vérité, creusé par l'histoire religieuse, demeuré entier: le roi est nu, et le 
demeure, même si on s'évertue à clamer qu'il est bien coiffé (avec la couronne des droits de l'Homme, par exemple).

Le prosélytisme laïc, pressé d'en finir avec le mensonge de l'aliénation, a en effet oublié que le dévoilement de la vérité n'est possible qu'à travers le surgissement de la question qui la porte, appelant une adéquation entre l'Être, tel qu'il serait en lui-même, et la représentation qu'on s'en fait. C'est donc dire que la vérité suppose d'abord une béance, un gouffre entre deux ordres de réalité qu'elle viendrait combler. Le propre du christianisme est précisément d'avoir "ontologisé " cette dualité en la dilatant à l'extrême, renvoyant dans deux ordres étrangers le monde terrestre de l'ici-bas et le monde divin de l'au-delà. Notons tout de suite que d'un point de vue heuristique, ce procédé avait l'immense avantage de reconnaître que la question de la vérité ne peut se poser que dans un horizon herméneutique, même si la sédimentation de la vérité dans le Livre allait permettre la constitution d'une bureaucratie du sens monopolisant la tâche de colmater la brèche. Opération d'ailleurs délicate s'il en est, puisque son éventuel succès aurait signifié l'auto-anéantissement de cette nouvelle élite.

Ce titanesque travail d'endiguement ne constitua évidemment, heureusement, qu'une réussite partielle. Il n'en a pas moins inauguré un rapport critique à la transcendance, certes déjà présent chez les Grecs, mais désormais situé dans un axe temporel où, à la suite de la révélation christique, c'est l'historicité même de notre rapport au monde qui commençait à être assumée, fut-ce partiellement. La reconnaissance de l'autonomie d'un domaine divin nourrissait en effet en retour l'idée d'une plénitude du monde terrestre jusque-là impensable. Cette autonomie de l'ici-bas, que le discours laïc situe dans l'ordre du donné, du factuel, reprend ainsi à son compte le dualisme chrétien, en en effaçant simplement l'un des termes. Les conséquences d'un tel geste sont immenses : c'est la nécessité même du détour de la société par la transcendance, pour s'appréhender comme réalité autonome, qui se trouve à être mise en cause. Du même coup, le politique, historiquement pensé comme la voie d'échappement à la mainmise du religieux sur la société, cesse d'être synonyme d'auto-institution de l'espace humain de l'être-ensemble. La laïcité, dont le développement est réputé aller de pair avec l'extension de l'autonomie de l'ordre humain, nourrit alors, au contraire, l'hétéronomie croissante de celui-ci : une nouvelle forme d'hétéronomie, à caractère systémique, plus radicale encore que son ancêtre religieux, qui ne pouvait faire l'économie de la réflexivité, fut-elle considérée comme une émanation divine.

Les laudateurs nostalgiques de la théocratie n'ont cependant aucune raison de se réjouir de la critique de la laịcité esquissée ici. Mon intention 
n'est pas de prendre position pour ou contre la laïcité, mais de chercher à comprendre ce que signifie aujourd'hui l'invocation plus ou moins militante qui en est faite. Car la laïcité a une histoire, qui ne peut être enfermée dans sa version française et républicaine, intimement liée au positivisme du XIX" siècle. Comme le mentionne si bien Michel Henry, "La mort de Dieu", le leitmotiv mélodramatique de la pensée moderne attribué à quelque percée philosophique audacieuse et repris en chœur par le psittacisme contemporain, n'est que la déclaration d'intention de l'esprit moderne et de son positivisme le plus plat » (HENRY 1996: 332-333). I1 faut replacer dans ce cadre la discussion sur la laïcité pour mesurer les apories sur lesquelles elle débouche et ouvrir d'autres perspectives, davantage soucieuses d'assurer une présence profane au monde qui soit néanmoins respectueuse de la nécessaire "métaphysique dans l'homme ", pour reprendre la belle expression de Maurice Merleau-Ponty.

\section{La laïcité dans les rets du positivisme}

Laîcité et positivisme : les deux concepts sont à ce point liés dans la tradition française qu'on en vient à désespérer des nombreux appels lancés aujourd'hui pour reconnaître le modèle d'avenir dans un républicanisme français daté, qui prend d'ailleurs l'eau de toutes parts. Car il faut bien le dire : la prétention universaliste du «modèle " français - face auquel nous devrions être particulièrement suspicieux au Québec, ne futce que parce qu'il est incapable de penser la question de la nation - s'inscrit au contraire dans le contexte d'une telle singularité historique (dans sa réponse plutôt que dans le problème auquel la France fut confrontée, ce qui explique qu'il puisse néanmoins prendre valeur d'exemplum) qu'il faut comprendre celle-ci pour mesurer la caducité de l'appel à la «solution» française.

On sait la place centrale qu'occupe la question de l'éducation dans le débat sur la laïcité en France depuis le dernier quart du XIXe siècle. Mais il est intéressant de constater à quel point le contexte épistémologique dans lequel cette transformation s'est opérée est presque toujours passé sous silence ${ }^{1}$. C'est pourtant lui qui permet de comprendre pourquoi la question de la laîcité va se cristalliser précisément à cette époque. Déjà,

1 Mentionnons tout de même la notable exception du livre de Louis LEGRAND, L'influence du positivisme dans l'oeuvre scolaire de Jules Ferry. Les origines de la laïcité (Paris, Librairie Marcel Rivière, 1961). Mais l'étude, centrée sur la question précise du rapport de Ferry au positivisme, ne procède pas à une analyse socio-historique critique de la signification du positivisme dans le nouveau rapport entre la transcendance divine et l'immanence terrestre, pas plus qu'il n'articule vraiment le rôle clé de l'éducation dans cette nouvelle économie du rapport à la transcendnce. 
dans la première moitié du siècle, Auguste Comte avait identifié trois grandes périodes historiques correspondant à autant de moments objectifs - de passages obligés - d'une histoire universelle que prétendait éclairer sa fameuse Loi des trois états. L'état théologique ou fictif (comportant luimême les phases du fétichisme, du polythéisme et du monothéisme), puis l'état métaphysique ou abstrait, apparaissaient comme un vaste préambule nécessaire à l'état positif ou réel, où "l'esprit humain renonce désormais aux recherches absolues qui ne convenaient qu'à son enfance, et circonscrit ses efforts dans le domaine, dès lors rapidement progressif, de la véritable observation, seule base possible des connaissances vraiment accessibles, sagement adaptées à nos besoins réels " (COMTE 1974 : 18).

C'est dans cet état positif que la question de la laïcité connaîtra la fortune que l'on sait. Le positivisme de Comte inaugure en effet, à travers la promotion d'une nouvelle posture épistémologique, une véritable pédagogie sociale. En éclairant des déterminations historiques désormais réputées objectivement identifiables, l'esprit scientifique vise à permettre à l'individu de se conformer à l'ordre objectif du monde. Une aporie loge ainsi au cœur du positivisme: pourquoi les normes fondant l'humanité devraient-elles faire l'objet d'un travail pédagogique, qui suppose nécessairement la subjectivité, si elles sont objectives? Pour dépasser, du moins en apparence, la difficulté (l'impossibilité) pour le sujet de se saisir lui-même comme une chose, le positivisme appréhende la dualité sujet/objet dans une perspective historique (ou développementale), au terme de laquelle la distance entre les deux pôles du rapport de connaissance sera abolie, à travers la réification de la société, comprise comme totalité objective. C'est donc d'une transformation radicale de la place du sujet dans le monde dont témoigne le positivisme. Et la laícité ne constitue que le versant politique de cette illusion épistémologique d'une possible appréhen. sion de la réalité sociale comme "chose". Mais de même que le projet de connaissance positiviste ne peut en son essence ouvrir qu'un intervalle historique (devant se terminer par la réification de la réalité sociale, saisissable seulement par le scientifique, ce nouveau « dieu " dont la capacité de demeurer à l'écart du réel, en le surplombant, demeure un impensé radical), la laïcité ne correspond qu'à un épisode transitoire qui ne peut déboucher, comme on le verra plus loin, que sur la légitimation d'un technocratisme aimant clamer son pluralisme, alors qu'il concourt de fait à la dissolution de la spécificité ontologique de la pratique.

En appréhendant la réalité sociale comme une totalité positive, Comte " annonce " en effet un nouveau rapport entre la théorie et la pratique dans le sillon duquel l'idée de laïcité pourra être creusée. Certes, l'autonomie du monde terrestre avait été pensée avant lui, au premier chef par les théoriciens du contrat social et, plus largement, les Lumières, qui fai- 
saient de la société un ordre purement formel, rationnel, ou à tout le moins rationalisable : c'est d'ailleurs pour cette raison que l'éducation avait acquis une telle importance à leurs yeux (qu'on songe par exemple à Locke et à Rousseau). Dans cette perspective, le rapport sujet/objet relevait d'un dualisme ontologique très clair: le sujet, pure réflexivité se constituant comme liberté à travers sa soumission au devoir-être de la Raison, s'opposait à l'objet, entendu comme pure chose soumise à des déterminations objectives, relevant de lois universelles identifiables par le sujet de Raison ${ }^{2}$. Il est clair que dans un tel cadre de pensée, la dimension normative de la société n'était pas chosifiée, comme elle le sera dans la perspective positiviste : elle était simplement refoulée, à travers sa projection dans un avenir à construire, assimilé au devoir-être de la Raison. Comme, sous les Lumières, le sujet se pense dans l'évidence d'une transparence à lui-même, la laïcité ne se pose pas en tant que telle comme problème. Guidé par une Raison qui se déploie en transformant en cadavres les normes traditionnelles, jugées irrationnelles parce que contingentes $^{3}$, le sujet finit cependant par s'épuiser dans une pure idéalité incapable de l'orienter dans le monde. C'est alors, et alors seulement, que la laïcité peut apparaître comme un enjeu central, parce qu'on lui prête la capacité de donner un contenu à une dynamique essentiellement négatrice. Mais elle ne peut que pousser à son paroxysme ce mouvement, en le tournant entièrement contre la religion, qui se trouve à subsumer en elle toutes les traditions sous la Tradition.

2 Si les Lumières ont tout misé sur l'idéalisme, il ne faut pas oublier qu'un autre courant, la Scottish Enlightenment (dans laquelle on peut entre autres situer des penseurs tels que Francis Hutcheson, David Hume, Bernard Mandeville, Adam Ferguson, William Robertson et, bien sûr, Adam Smith), prenait une direction radicalement opposée, et en quelque sorte complémentaire. C'est principalement de cette tradition de pensée anglo-saxonne (mais pas uniquement d'elle, comme en témoigne le courant physiocrate en France) que sortira l'économie politique, qui a fini par asseoir l'immanence du monde terrestre sur l'objectivité des rapports intéressés que les individus sont réputés devoir nécessairement nouer entre eux, aiguillés qu'ils sont par leurs besoins. Ce versant "matérialiste" des Lumières, qui appréhende l'histoire humaine comme une histoire naturelle, se déploie comme le négatif de son pendant idéaliste, qui culmine avec Kant et son historicisation de la théodicée. Historiquement, le positivisme du $\mathrm{XIX}^{\mathrm{e}}$ siècle constitue une réponse à la prétention économiciste de réduire la société à une somme de rapports inter-individuels, en reconnaissant au contraire la société comme une réalité sui generis - et non plus simplement formelle pouvant être saisie dans sa positivité.

3 Voir à ce sujet Michel FREITAG, "La Raison contre les raisons », Société 2, automne 1987. 
La spécificité française de la lä̈cité, indissociable du positivisme ${ }^{4}$ (et, partant, de l'éducation), n'épuise cependant pas, loin s'en faut, la réalité historique de la revendication laïque : la France de Jules Ferry n'a pas le privilège d'avoir initié la question de la laïcité, même si elle en a déplacé l'enjeu. Dans un ouvrage classique trop souvent oublié, La naissance de l'esprit lä̈que au déclin du Moyen Âge, Georges de Lagarde rappelle ainsi que la distinction entre les clercs et les laïques se retrouve déjà dans les premières églises chrétiennes. Il note d'emblée que "la religion nouvelle n'avait pas tardé à créer une différence bien tranchée entre le peuple des fidèles «enseignés» [...] et les divers membres du sacerdoce [...] En ce sens, la distinction des "clercs " et des "lä̈ques" existe encore dans presque toutes les églises chrétiennes. Elle oppose le troupeau au pasteur, les brebis au berger, les fidèles à la hiérarchie » (DE LAGARDE 1956: VIII).

\section{Le christianisme comme pédagogie}

Cette distinction entre les "enseignants " et les "enseignés " pointe déjà vers la dimension pédagogique du christianisme, ce qui indique bien que le rapport entre laïcité et éducation n'a pas attendu le XIXe siècle pour se développer. Lessing l'avait d'ailleurs bien compris, qui écrivait déjà, en 1780 , dans L'éducation du genre humain, que

la Révélation est au genre humain ce que l'éducation est à l'individu. L'éducation est une révélation qui a eu lieu chez l'individu, et la Révélation est une éducation qui a lieu encore chez le genre humain. Je ne veux pas examiner ici s'il peut être utile pour la pédagogie d'envisager l'éducation de ce point de vue. Mais, en théologie, se représenter la Révélation comme une éducation du genre humain est à coup sûr très utile pour résoudre bien des difficultés (LESSING 1995 : 11-12).

Je noircis évidemment le trait. Le rapport entre la laïcité française et le positivisme n'est bien sûr pas aussi unilatéral. Je n'ignore pas qu'à la même époque, le néo-kantisme tente de sortir le sujet transcendantal kantien des vapeurs de son idéalité, pour le réinscrire dans la chair de l'histoire. C'est d'ailleurs dans une perspective kantienne ou néo-kantienne que sont le plus souvent pensées aujourd'hui, en France, les questions de la république et de la laïcité. Mais il faut bien voir que cette perspective n'échappe pas davantage que le positivisme à l'incapacité de ramener la transcendance - autrement que comme forme vide trouvant maintenant dans la communication, par exemple, une façon de se donner " un air " de concrétude - dans le monde de l'ici-bas. C'est davantage dans un horizon hégélien, cependant dégagé de toute entéléchie, que peut être envisagée la transcendance mondaine (pour ne pas dire laïque), logeant dans le rapport entre le sujet et l'objet, dans son développement historique contingent, plutôt que dans l'un ou l'autre de ses pôles. 
Dans une perspective historique et sociologique, on pourrait ajouter que cette façon de concevoir la Révélation est peut-être celle qui permet le mieux de saisir la signification historique du christianisme et, de là, la revendication laïque qui en est sortie.

Le christianisme a le premier permis l'adoption d'une posture réflexive dans un monde désormais reconnu dans sa dimension historique - dans l'attente du Jugement dernier. Certes, cette position n'y fait pas elle-même l'objet d'un travail critique, si ce n'est dans les marges ${ }^{5}$. La transmission d'une vérité ayant pour tous les membres de la chrétienté une charge normative très contraignante s'appuie en effet sur un socle dogmatique. Mais l'éducation chrétienne a toujours postulé dans son dessein même l'existence d'une intériorité subjective dont il lui fallait en quelque sorte assurer l'édification. Le propre de la modernité a été d'universaliser - du moins idéologiquement - l'accès à une réflexivité critique (ou de second niveau). Ce faisant, la modernité reprenait à son compte, tout en la déplaçant, une scission au sein de l'Être, léguée par le christianisme : c'est ce qui lui a permis de distinguer les questions de la « nature de l'Être " et de la "nature de la vérité », quitte à les faire coïncider dans une vérité de l'Être à laquelle la Raison sera présumée assurer l'accès.

L'abord du christianisme comme pédagogie n'implique nullement qu'aucune remise en cause de son enseignement ne soit possible. Il exige cependant que soit reconnue la primauté du chemin (du questionnement) sur la destination (la vérité ?), et peut certainement contribuer à éviter que ne se referme le gouffre évoqué plus haut. Ce qui se joue dans la reconnaissance du statut pédagogique du christianisme n'est en fait rien d'autre que la façon dont nous saurons - ou non - collectivement repenser notre rapport à la transcendance, au lieu d'accentuer l'actuel mouvement de sa négation technique dans une indifférenciation du "vrai " et du "faux", mais aussi du sujet et de l'objet, du déterminisme et de la liberté (qui tend à se réduire au hasard), de l'État et de la société civile, etc. Tel est le véritable enjeu de l'admission d'une dette collective à l'égard du christianisme, comme événement historique, que la revendication laïque ne semble pas capable de cerner, parce qu'elle se place sur un terrain où ce problème ne peut tout simplement pas être abordé.

Il ne s'agit pas davantage ici de passer l'éponge sur l'autoritarisme qui traverse l'histoire chrétienne, déchirée par le conflit entre le pouvoir spirituel et le pouvoir temporel. Mais à trop vouloir approcher le christianisme à travers ce prisme, on finit par le déformer, jusqu'à oublier que

5 N'est-ce pas là la spécificité de la théologie, critique en son essence même, y compris chez ses représentants les plus conservateurs? 
c'est lui qui a permis d'aborder la question du fondement de l'autorité dans un monde désormais historique. C'est cette question de l'autorité qui se trouve maintenant indirectement soulevée par l'invocation de la laïcité. Indirectement, parce qu'elle est rarement assumée comme telle, l'autorité étant d'emblée renvoyée dans l'orbite "réactionnaire" de l'assujettissement et de l'oppression. Comme si le projet émancipateur de la modernité, en tant qu'auto-fondation assumée de la société, était passé par une abolition de l'autorité plutôt que par son abstraction dans la figure impersonnelle et à prétention universaliste de la Raison!

Le rapprochement fait ici entre l'approfondissement de la réflexivité médiatisée par le rapport à l'Autre, condensé dans la figure divine - et le christianisme implique-t-il pour autant que la modernité n'ait au fond constitué qu'une "théologie déguisée " (DUPRÉ 1976:141), dépourvue d'idées originales, dans la mesure où elle n'aurait fait que procéder à un transfert de certaines catégories chrétiennes dans le domaine laïc? C'est en tout cas ce que défendent - avec plus ou moins de zèle - les tenants de la thèse de la sécularisation. Karl Löwith, l'un de ses plus célèbres partisans, l'affirme sans ambages: "We of today, concerned with the unity of universal history and with its progress toward an ultimate goal or at least toward a "better world", are still in the line of prophetic and messianic monotheism; we are still Jews and Christians, however little we may think of ourselves in those terms" (LÖWITH 1949: 19).

Cette approche a l'immense mérite de poser d'entrée de jeu la question du rapport laïc/religieux dans une perspective historique, fut-ce sur le mode du transfert, ou de l'épuisement d'un type de rapport - à soi, à autrui et à la nature - essentiellement religieux au monde. Il n'est pas certain pour autant qu'elle précise véritablement les enjeux qui sont les nôtres aujourd'hui. La thèse d'une transposition au profane d'idées d'origine sacrale ou religieuse est certes séduisante, et certainement valable dans bien des cas ${ }^{6}$. Elle présuppose cependant déjà un dualisme entre la transcendance divine et l'immanence terrestre, et se situe ainsi en aval

6 Encore que dans ce cas comme dans bien d'autres, la modération soit de mise. Il n'est ainsi pas du tout certain que Löwith favorise une meilleure compréhension du communisme et du fascisme lorsqu'il affirme, dans un chapitre consacré à Joachim DE FLORE (1131-1202): "The revolution which had been proclaimed within the framework of an eschatological faith and with reference to a perfect monastic life was taken over, five centuries later, by a philosophical priesthood, which interpreted the process of secularization in terms of a "spiritual" realization of the kingdom of God on earth [...] The third dispensation of the Joachites reappeared as a third International and a third Reich, inaugurated by a dux or a Führer who was acclaimed as a savior and greeted by millions with Heil! " (LÖwITH 1949 : 159). 
du cœur philosophique de la question du rapport à la transcendance, que pose aujourd'hui (sans clairement le savoir ?) la laïcité. À cet égard, plutôt que de parler d'un transfert d'éléments sacraux vers le profane pour caractériser la modernité, il vaudrait peut-être mieux parler, plus simplement, de l'approfondissement de la césure à travers laquelle le sujet et l'objet peuvent naître au monde, au point de les renvoyer dans deux sphères ontologiques radicalement disjointes.

\section{Matérialisme chrétien ou idéalisme profane?}

Dans Le désenchantement du monde. Une histoire politique de la religion, Marcel Gauchet a bien montré comment le christianisme procède d'une séparation tellement radicale de l'ici-bas et de l'au-delà qu'elle a finalement entraîné la sortie de la société de la religion. Cette extériorité radicale des deux mondes, entre lesquels l'homme-Dieu jeta un pont singulier, constitue la toile de fond de l'histoire du christianisme, marquée par d'innombrables tentatives de refermer dogmatiquement cette distance infinie : c'est en quelque sorte à rebours de son fondement que le christianisme s'est ainsi déployé, en voulant recouvrir le pouvoir temporel par le pouvoir spirituel. Mais c'est précisément l'invocation de l'autonomie de la transcendance divine, censée justifier sa domination sur le monde terrestre, qui a permis, a contrario, de reconnaître l'immanence de l'ici-bas. Dans cette optique, la déliaison des deux mondes, dont le crédit est souvent porté au compte du protestantisme (c'est le cas par exemple chez Max Weber), doit être vue comme une réalité - à actualiser - présente dès le début du christianisme. L'histoire de celui-ci, loin d'être portée par quelque déterminisme, est bien sûr tissée dans une contingence prenant souvent les allures d'une dénégation de cette potentialité originelle. Elle n'en a pas moins mené à une configuration de notre rapport à la transcendance qui fait de ce qu'on appelle maintenant le laïc et le religieux les deux faces d'un même rapport. Le corollaire de cette histoire est clair : le rapport prime sur les pôles que son extension rend lisible. C'est toute la portée d'une telle leçon qu'il nous faut collectivement saisir aujourd'hui, si l'on veur éviter que l'histoire contingente qui nous porte, et qui a marqué notre inscription de plus en plus réfléchie dans le monde, finisse par échouer sur les rives d'un réel libéré - ou plutôt pillé - de toute capacité de faire retour sur lui-même.

L'expansion moderne de l'espace d'autonomie de l'être humain s'est en effet appuyée sur une représentation tellement abstraite de ce dernier qu'elle a fini par le renvoyer quasiment hors du monde, dans une pure idéalité (l'être de Raison) face à laquelle la réalité - assimilée à la simple résultante physique de rapports de forces - paraît désormais dénuée de toute présence à elle-même. La profondeur de ce schéma est aujourd'hui à 
ce point achevée que je ne m'étonne plus de me faire taxer d'idéaliste moi qui suis agnostique - par des chrétiens, fussent-ils progressistes, qui semblent ainsi avoir renoncé à ramener l'idéalité dans ce monde qui est le nôtre, ce qui aurait pour conséquence de nous obliger collectivement à assumer nous-mêmes la définition des normes concrètes (et non plus perdues dans une abstraction négatrice ayant fait son temps) devant baliser l'espace de notre être-ensemble?.

L'aval qui se trouve ainsi donné, par des croyants, au matérialisme le plus plat, procède d'une mécompréhension de la spécificité de la dynamique moderne, que Gauchet lui-même me paraît oublier dans un schéma historique pourtant très suggestif, où l'histoire occidentale se lit comme une réduction continuelle de l'altéritê ${ }^{8}$. Mais la modernité ne marque pas tant une réduction de la mise à l'écart du fondement du monde, que

7 Ainsi entendu, l'idéalisme n'implique nullement la négation de l'existence de " rapports de forces » au sein de la société. Il permet cependant de resituer ceux-ci dans le cadre des médiations institutionnelles qui en constituent la condition de possibillité. Les " purs " rapports de forces n'existent que dans les livres de physique, et à vouloir appréhender la société à travers le filtre de cette dernière, on comprend qu'il faille condenser la subjectivité - l'intentionnalité - dans une idéalité transcendante (Dieu), ultime, voire unique source du sens de ce qui est. Pourtant, même le croyant - sauf à nier sa propre existence subjective, et donc sa foi - ne peut accorder à Dieu un tel monopole de la subjectivité. Et le rapport subjectif qui le lie à autrui ne peut, par quelque alchimie, être réduit à une réalité objective. Cela n'empêche pas que la société puisse apparaître, tant aux yeux du croyant que du non-croyant, comme une réalité de l'ordre du donné. Mais c'est précisément le rôle de la pensée critique - à laquelle la foi ne peut être déclarée étrangère par principe, puisqu'elle suppose elle-même un minimum de distance par rapport à ce qui est - de montrer que l'idée d'objectivité voile en fait l'objectivation historique de la société, qui n'existe qu'à travers la reproduction individuelle de pratiques médiatisées symboliquement et institutionnellement. Autre façon de dire que la subjectivité ne se comprend que comme inter-subjectivité, impliquant une verticalité (une transcendance) à travers laquelle les membres d'une société peuvent se reconnaître, à défaut - du moins pour les non-croyants - de reconnaître Dieu. Je ne nierai certainement pas la fragilité ontologique de cet ordre de réalité, toujours susceptible de sombrer dans une inadéquation entre les pratiques particulières et le cadre signifiant dans lequel elles tentent de s'inscrire. Mais à défaut de pouvoir dire si cette fragilité suffit à expliquer la "naissance " de Dieu, je dirai qu'elle résonne aujourd'hui comme un appel à la responsabilité collective de la reconnaître comme telle. Cela ne pose plus tant la question de notre rapport à Dieu, qu'il soit réel ou fictif, que celle du rapport à nous-mêmes, à travers le nécessaire détour d'une représentation commune qui puisse faire sens pour chacun de nous parce qu'elle prend valeur de projet.

8 Le lecteur consultera à ce sujet l'article de Michel FrEITAG sur “ La naissance du politique dans les sociétés traditionnelles ". 
Gauchet pose à son zénith dans les sociétés primitives, que son abstraction dans un sujet rationnel, sur les épaules duquel est désormais reportée la fondation de la société, réalité formelle mais non moins réelle dont il lui incombe alors d'assurer explicitement la reproduction. Pleinement assumée comme réalité idéelle intra-mondaine dans la modernité, on comprend que la société pose alors avec insistance la question de la légitimité du pouvoir politique qui, à ce moment seulement, peut commencer à se déployer de façon autonome. D'où l'exemplarité du cas français, où, contrairement à l'Angleterre, pour ne nommer qu'elle, la question de la légitimité s'est posée avec d'autant plus d'insistance que le Royaume a tardé à reconnaître cet espace politique autonome, ce qui a appelé sa radicalisation idéologique, qui allait permettre d'assumer pleinement le virage vers un avenir à ériger, en rupture avec un passé à subir.

Le problème sur lequel allait cependant rapidement se buter ce partage des eaux entre la subjectivité et l'objectivité - et auquel le positivisme a tenté sans succès de répondre - demeure entier aujourd'hui. Loin de loger dans l'inachèvement de la modernité, il consiste plutôt dans l'incapacité de celle-ci de définir des normes collectives ayant à la fois un caractère concret et réfléchi. Le travail du négatif spécifiant la modernité s'appuyait en effet sur une représentation abstraite du sujet incapable de penser son inscription concrète dans le monde autrement que sur le mode de la «table rase ». C'est donc cette vision du sujet qu'il nous faut maintenant repenser, non en rompant avec notre histoire, d'une façon moderne, mais en reconnaissant que cette histoire contingente - qui implique d'en finir avec l'idée d'un quelconque déterminisme - fait partie de notre Être.

À cet égard, la laïcité, parce qu'elle reprend sur un mode d'opposition la question de notre rapport religieux au monde, ne constitue pas à mes yeux une voie d'avenir. Cela ne signifie pas pour autant qu'il faille donner le blanc-seing (ou la bénédiction) à la prolifération contemporaine des mouvements religieux plus ou moins fondamentalistes ou "nouvelâgistes ». C'est au contraire par la remise en cause d'un schéma binaire partageant la réalité en un monde idéel (transcendant) et un monde objectif (immanent, terrestre) que l'écueil d'un irrémédiable no man's land normatif - avec le vide identitaire et l'écroulement psychique qu'il implique - pourra être évité. Sans vouloir jouer les prophètes de malheur, il faut néanmoins constater que la reconnaissance du problème - sans même parler de solutions - s'avère d'autant plus urgente que le dépassement de ce dualisme ontologique est déjà largement entamé, mais d'une façon technocratique, qui tend à l'indifférenciation radicale du sujet et de l'objet dans un même magma "environnemental " tendant à évacuer toute réflexivité. Comme si la réalité humaine pouvait être totalement délestée du sentiment qu'elle habite un monde, qu'elle est ce monde, avec 
ce que cela suppose comme épaisseur ontologique pour le sujet, impensable sans un minimum de «quant-à-soi ».

\section{Pour une nouvelle version de la parousie}

Pierre Legendre résume bien l'illusion qui nourrit maintenant l'Occident :

Les savants et les gestionnaires sont préposés, par les sociétés occidentales, à l'entreprise d'éliminer le mystère et la tragédie [...] Le Management est porté par la pensée occidentale qui dit: Dieu est mort, le pouvoir est injuste, l'industrie sera le gouvernement des choses [...] Le Management annoncerait-il un monde délivré de l'Abîme, affranchi de la tragédie: pouvoir de l'homme sur l'homme, guerre des sexes et roman de familles? Un monde vient, enfin géré, simplement géré, la politique devenue une technique, et la tragédie liquidée comme on renonce à l'absurde. Ainsi croyons-nous, en croyants d'aujourd'hui, pour vivre et survivre (LEGENDRE $1996: 27$ ).

La revendication à la laïcité, même habitée des meilleurs intentions du monde et nourrie d'appels au pluralisme, ne me paraît pas pouvoir marquer un terme à la situation décrite par Legendre. Simplement parce qu'elle est à côté du problème. Il ne suffit pas de prêcher le pluralisme pour rompre avec la logique gestionnaire. Quand la question de la légitimité de l'ordre social tend à devenir périmée, parce que n'est même plus pensée l'exigence du détour de la représentation comme condition de possibilité de l'existence de la société, le pluralisme devient le simple slogan d'un ersatz de légitimation (de moins en moins requise) pour une laïcité noyée dans la gestion technocratique du social. Reflet d'une réalité " objective", plutôt que visée idéelle subsumée sous une transcendance pleinement assumée, le pluralisme constitue l'une des chevilles ouvrières de l'État gestionnaire, "devenu le régulateur des joutes "sectorielles" entre des intérêts partiels, et le mat de cocagne d'une foire aux droits et privilèges particularistes »(SIMARD $1983: 211^{9}$ ). Dans cette optique, la

9 Il est "amusant" de remarquer que les appels au pluralisme, lorsqu'ils sont simplement drapés de la neutralité axiologique, débouchent sur l'exact inverse de la tolérance, et nourrissent en fait ce qu'on pourrait appeler "la dictature du réel ". Quand, au nom de l'égalité entre les êtres humains, l'ordre symbolique - à travers lequel nous «respirons» - se trouve aplati, déstructuré, c'est la possibilité même de faire sens qui se trouve mise à mal. Dans un monde ainsi "libéré " des balises (en soi réactionnaires?) pouvant orienter le sujet, c'est le fait qui finit par prendre, aux yeux de ce dernier, le statut de norme. Historiquement, la constitution du marché, qui tend à réduire progressivement toute action au plus petit commun dénominateur de l'intérêt, réputé constituer son ultime fondement, a fortement contribué à cette réification des 
laïcité, en consacrant idéologiquement - comme si besoin était - l'«horizontalisation" du social, montre son impuissance à mobiliser la réflexion pour permettre le dépassement d'un dualisme ontologique incapable de saisir que la théorie et la pratique sont au fond indissociables. Qu'est-ce à dire? Simplement que la modernité a condensé tout le moment réflexif de la pratique dans la théorie, qui se trouvait ainsi renvoyée dans une orbite transcendantale, privant du même coup le sujet concret de sa dimension idéelle. La table était ainsi mise pour que la pratique, délestée de tout rapport à soi, soit happée par les experts en tous genres. Dans le texte introductif du premier numéro de la revue Libre, Claude Lefort décrivait déjà ce qui constitue aujourd'hui une réalité massive.

L'homme en vient à se rapporter au travail par le médium de la technologie, à son désir par le médium de la sexologie, à la nourriture par celui de la diététique, à l'enfant ou au nourrisson par celui de la pédagogie ou de l'esclavage rationnel, à la nature par celui de l'écologie, d'une façon générale à son corps, sa vie et les autres par mille petits modèles scientifico-psychologiques ou sociologiques. Un fantastique projet d'intimidation soutient ce discours de connaissance qui contraint le grand nombre à se soumettre aux représentations de l'expert, mieux : à les intérioriser sous peine soit de n'être rien, soit de se percevoir soi-même comme a-social ou déviant ou déchet (1977:20).

Nous en sommes là, et la laïcité n'y changera rien, incapable qu'elle est d'aborder, autrement que par la négative, la question des normes substantielles - déterminées de façon critique - que notre histoire exige désormais. C'est, étrangement, par un retour aux sources de l'abîme qui est le nôtre que nous pourrons peut-être reprendre en main notre destin. La médiation christique, annonce de la parousie, a permis de déplier un espace temporel à l'intérieur duquel l'Occident a pu entrer dans un rapport critique au monde. C'est sur elle qu'il nous faut tabler, et sur sa tentative de définir substantiellement ce que peut vouloir dire concrètement quelque chose comme le "bien ". Dans notre monde profane, cet héri-

normes. On se retrouve ainsi dans une situation où le pluralisme est aujourd'hui fréquemment conjugué au même temps que le marché. Le résultat est clair : toute visée d'auto-compréhension de soi de la société est assimilée à un projet totalitaire et doit ainsi s'effacer derrière la recherche opératoire d'une capacité croissante d'agir immédiatement sur le réel. La médiation symbolique de notre rapport au monde se délite dans un succédané de sens, où le cliché et le slogan apparaissent désormais comme quelque chose de "cool", alors qu'en oubliant toute visée de signification, avec ce que cela suppose comme détour réflexif, ils participent d'un mouvement se situant aux antipodes de toute prétention à l'autonomie du sujet. 
tage doit être étendu du côté de l'œecuménisme - entendu dans le sens le plus large possible - afin que nous puissions commencer à penser notre avenir, plutôt que de craindre qu'il nous tombe dessus. En dignes héritiers de la modernité conscients des limites de celle-ci, nous pourrons ainsi donner un contenu significatif à notre présence au monde. Cela passera par la reconnaissance de l'historicité de notre être, dont les contingences, loin d'être accessoires, représentent plutôt un trésor qu'il nous faut chérir, en le partageant avec les trésors d'autres traditions également appelées à cette réflexion " œcuménique ${ }^{10}$ ".

Au lieu d'attendre la parousie devant effacer la dualité ontologique consacrée par le christianisme, il nous incombe désormais de relier nousmêmes deux ordres de réalités dont la séparation historique a constitué un formidable ferment pour la conscience humaine. "Apprendre à prier ", disait Schönberg. Cela peut vouloir dire, aujourd'hui, se réapproprier un monde abandonné aux puissances objectales. Cela doit vouloir dire, aussi, qu'il faut cesser de voir un fantôme totalitaire tapi derrière la volonté de nous donner nous-mêmes un destin commun, ce qui suppose d'appréhender la société comme une totalité sui generis. Cela ne donne pas de réponse. Et cela exige beaucoup de sagesse, une sagesse informée par un temps historique qui dure à travers nous, et qu'il nous faut apprendre à cultiver pour ce qu'il est : la richesse de l'inutile, qui en fait toute la beauté.

\section{Bibliographie}

COMTE, Auguste. 1974 (1844). Discours sur l'esprit positif. Paris, Vrin, $172 \mathrm{p}$.

DUPRÉ, Louis. 1976. "La sécularisation et la crise de notre culture ", dans Herméneutique de la sécularisation. Paris, Aubier, pp. 141-152.

FREITAG, Michel. 1988. «La Raison contre les raisons », Société 2: 181 . 230.

FREITAG, Michel. 1989. "La genèse du politique dans les sociétés traditionnelles", Société $6: 41-122$.

GAUCHET, Marcel. 1985. Le désenchantement du monde. Une histoire politique de la religion. Paris, Gallimard, $306 \mathrm{p}$.

HENRY, Michel. 1996. C'est moi la vérité. Pour une philosophie du christianisme. Paris, Seuil, 347 p.

10 Au crédit de l'éducation laïque française, on peut d'ailleurs noter un pas dans cette direction, à travers l'accent qu'elle a mis sur l'importance de la littérature, dont il est inutile de dire à quel point elle s'inscrit dans le sens de ce que je défends ici. 
LAGARDE, Georges de. 1956. La naissance de l'esprit lä̈que au déclin du Moyen Âge. I- Bilan du XIII siecle. Louvain-Paris, Nauwelaerts, 217 p.

LEFORT, Claude. 1977. "Maintenant ", Libre 1:3-28.

LEGENDRE, Pierre. 1996. La fabrique de l'homme occidental. Paris, Mille et une nuits/Arte, $55 \mathrm{p}$.

LESSING, Gotthold Ephraïm. 1995 (1780). "L'éducation du genre humain ", dans Le crépuscule des Lumières. (Tavoillot, Pierre-Henri, dir.), Paris, Cerf, pp. 11-29.

LÖWITH, Karl. 1949. Meaning in History. Chicago, University of Chicago Press, 259 p.

MERLEAU-PONTY, Maurice. 1966. "La métaphysique dans l'homme », dans Sens et non-sens. Paris, Nagel, pp. 145-172.

SIMARD, Jean-Jacques. 1983. "L'œil-de-dieu au beurre noir: la société contre le sens et la religion à l'école ", dans Confessionnalité et pluralisme dans les écoles du Québec. (DENAULT, Bernard et OUELLET, Fernand, dir.), Montréal, Les Cahiers de l'ACFAS, pp. 210-233. 